\title{
Estudo biomecânico de diferentes dispositivos de fixação femoral na reconstrução do ligamento patelofemoral medial em joelhos de suínos*
}

\section{Biomechanical Study of Different Femoral Fixation Devices in the Reconstruction of the Medial Patellofemoral Ligament in Porcine Knees}

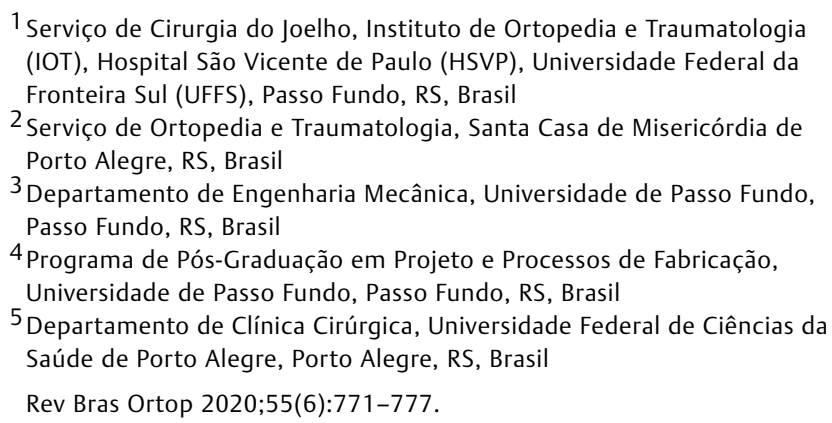

${ }^{1}$ Serviço de Cirurgia do Joelho, Instituto de Ortopedia e Traumatologia (IOT), Hospital São Vicente de Paulo (HSVP), Universidade Federal da Fronteira Sul (UFFS), Passo Fundo, RS, Brasil

${ }^{2}$ Serviço de Ortopedia e Traumatologia, Santa Casa de Misericórdia de Porto Alegre, RS, Brasil

${ }^{3}$ Departamento de Engenharia Mecânica, Universidade de Passo Fundo, Passo Fundo, RS, Brasil

${ }^{4}$ Programa de Pós-Graduação em Projeto e Processos de Fabricação, Universidade de Passo Fundo, Passo Fundo, RS, Brasil

${ }^{5}$ Departamento de Clínica Cirúrgica, Universidade Federal de Ciências da Saúde de Porto Alegre, Porto Alegre, RS, Brasil

Rev Bras Ortop 2020;55(6):771-777.

\author{
Resumo \\ Palavras-chave \\ - fenômenos \\ biomecânicos \\ - ligamentos \\ articulares \\ - modelos animais \\ - articulação \\ patelofemoral \\ - técnicas de sutura \\ - dispositivos de \\ fixação ortopédica
}

Endereço para correspondência Osmar Valadão Lopes Jr., MD, MSc, Rua Uruguai, 2050, Passo Fundo, RS 99010-112, Brasil (e-mail: osmarlopesjr@gmail.com; pesquisa2@hsvp.com.br).

\footnotetext{
Trabalho realizado no Hospital São Vicente de Paulo (HSVP-RS), Instituto de Ortopedia e Traumatologia de Passo Fundo (IOT-RS) e Laboratório de Bioengenharia, Biomecânica e Biomateriais da Universidade de Passo Fundo (UPF).
}

recebido

08 de Outubro de 2019

aceito

12 de Dezembro de 2019
Objetivo Avaliar diferentes dispositivos de fixação femoral na reconstrução do ligamento patelofemoral medial para comparar sua eficácia quanto à força de fixação até a falha em joelhos suínos.

Métodos Foram ensaiados 30 joelhos de suínos subdivididos em 3 grupos de 10 joelhos. Os enxertos retirados foram dissecados de tendões extensores das patas dos suínos. Cada grupo teve o enxerto fixado ao fêmur com parafuso de interferência, âncora, ou tenodese no tendão adutor. Os 3 métodos foram submetidos à testes biomecânicos utilizando uma máquina universal de ensaio de tração com uma velocidade de $20 \mathrm{~mm} / \mathrm{min}$.

Resultados Verificamos que a média mais elevada da resistência linear sob tração lateral $(185,45 \pm 41,22 \mathrm{~N})$ ocorreu no grupo 1: "fixação por parafuso," seguido do grupo 2: "fixação por âncora" (152,97 $\pm 49,43 \mathrm{~N})$, e a média foi menor no grupo 3: "fixação por tenodese" $(76,69 \pm 18,90 \mathrm{~N})$. Para a margem de erro fixada (5\%), comprovou-se a diferença significativa entre os grupos $(p<0,001)$ e também através dos testes de comparações múltiplas (entre os pares de grupos) verificou-se a ocorrência de diferenças significativas. A variabilidade expressada por meio do 


\section{Abstract}

\section{Keywords}

- biomechanical phenomena

- joint ligaments

- animal models

- patelofemoral joint

- suture technique

- orthopedic fixation devices coeficiente de variação mostrou-se reduzida, já que a referida medida foi inferior a $33,3 \%$.

Conclusão $\mathrm{O}$ uso de parafusos de interferência no túnel ósseo de joelhos porcinos é suficientemente forte para fixação femoral na reconstrução do ligamento patelofemoral medial, assim como a fixação com âncoras montáveis com fio de alta resistência. Entretanto, a tenodese no tendão adutor mostrou-se frágil para essa finalidade.

Objective To evaluate different femoral fixation devices for medial patellofemoral ligament reconstruction and compare their effectiveness regarding fixation strength up to failure in porcine knees.

Methods Thirty porcine knees were used, divided into three groups of 10 knees. The removed grafts were dissected from the extensor tendons of porcine feet. In each group, the graft was fixed to the femur with an interference screw, an anchor, or adductor tenodesis. The three methods were subjected to biomechanical tests using a universal Tensile testing machine at a speed of $20 \mathrm{~mm} /$ minute.

Results The highest average linear resistance under lateral traction occurred in group 1, "screw fixation" $(185.45 \pm 41.22 \mathrm{~N})$, followed by group 2, "anchor fixation" $(152.97 \pm 49,43 \mathrm{~N})$; the lower average was observed in group 3, "tenodesis fixation" $(76.69 \pm 18.90 \mathrm{~N})$. According to the fixed error margin $(5 \%)$, there was a significant difference between groups $(p<0.001)$; in addition, multiple comparison tests (between group pairs) also showed significant differences. Variability was small, since the variance coefficient was lower than $33.3 \%$.

Conclusion Interference screws in bone tunnels and mountable anchors fixation with high resistance wire are strong enough for femoral fixation in porcine medial patellofemoral ligament reconstruction. Adductor tenodesis, however, was deemed fragile for such purpose.

\section{Introdução}

A anatomia e as propriedades biomecânicas do ligamento patelofemoral medial (LPFM) são descritas em diversos trabalhos. $^{1-8}$ O LPFM está situado na segunda das três camadas da região medial, juntamente com o ligamento colateral medial e tem trajeto transversal da patela ao fêmur. No fêmur, insere-se posterior e proximalmente ao epicôndilo medial e distal e anteriormente ao tubérculo do adutor. Seu comprimento médio varia entre 53 e $55 \mathrm{~mm}$ e sua largura entre 3 e $30 \mathrm{~mm} .{ }^{4} \mathrm{O}$ LPFM é um dos principais estabilizadores mediais da patela, suas lesões (na maioria das vezes na inserção femoral) estão associadas às luxações de patela. ${ }^{1-3}$ O LPFM é frequentemente reconstruído em pacientes com instabilidade recorrente da patela, mostrando bons resultados em estudos clínicos ${ }^{1,3,5,7}$ e é o principal limitador do deslocamento lateral da patela, direção em que ocorrem a maioria das luxações, contribuindo com $60 \%$ da força de restrição na lateralização em flexão de $20^{\circ}$ do joelho. ${ }^{4} \mathrm{~A}$ reconstrução do LPFM foi descrita primeiramente por Gomes et al. ${ }^{1}$ e é realizada com o objetivo de restaurar a estabilidade patelar, obtendo desfechos favoráveis. ${ }^{1,3,5,7}$

A reconstrução do LPFM é frequentemente realizada isoladamente quando a morfologia óssea é normal. Nas situações de displasia troclear e/ou patela alta, o LPFM pode desempenhar um papel ainda maior na restrição biomecânica do que quando a fossa troclear e a altura da patela estão normais. ${ }^{6}$

Muitas técnicas cirúrgicas foram desenvolvidas para tratar a instabilidade patelar: enxerto livre fixo em canais ósseos, enxerto livre fixado com âncoras, e enxerto livre suturado ao periósteo. ${ }^{7}$ Atualmente não existe opção padrão-ouro para fixação femoral, sendo a técnica mais frequentemente utilizada o túnel com parafuso de interferência e, em alguns casos, as âncoras com sutura. ${ }^{9}$

A fixação por tenodese é melhor indicada na reconstrução de LPFM em esqueletos imaturos e para evitar a sutura transtendão. ${ }^{10} \mathrm{~A}$ técnica de tenodese do enxerto no tendão do músculo adutor magno realizada em jovens tem a vantagem de não causar qualquer dano à fise aberta no fêmur, prevenindo o fechamento prematuro da mesma, podendo causar deformidade angular. ${ }^{11}$

As âncoras permitem a inserção direta de implantes (sem necessidade de perfuração, rosqueamento, ou pré-perfuração) com o uso de ponta de auto-inclusão, bem como aperfeiçoamento e tensionamento de suturas individuais. ${ }^{12}$ Foram demonstrados bons escores funcionais pela escala de Kujala em pacientes nos quais foram usadas âncoras de titânio para fixação femoral. Como apenas uma âncora de titânio é aplicada no fêmur, este procedimento é relativamente rentável; além disso, a utilização de muitos implantes para estabilizar o enxerto aumenta o risco de dor e inflamação local. ${ }^{11}$ 
Sabe-se que o material de fixação proeminente na parte medial do côndilo femoral medial pode também causar irritação local e restringir potencialmente o movimento. Até mesmo na ausência de um material proeminente, um túnel no côndilo femoral medial pode ser a fonte de dor refratária. ${ }^{7-9}$ A dor e a rigidez também podem estar relacionadas à lesão subjacente das superfícies articulares do compartimento patelofemoral ou também com o mau posicionamento do túnel. ${ }^{6-9}$

Deve-se também estar ciente de que qualquer material de síntese na borda da patela ou no lado medial do côndilo femoral pode tornar-se proeminente uma vez que o inchaço cirúrgico desapareça. Os pacientes podem tolerar menos o desconforto nestas áreas, solicitando a remoção do material. ${ }^{9}$

O presente trabalho visa testar e mensurar as propriedades biomecânicas de três diferentes métodos de fixação do enxerto no côndilo femoral medial, na reconstrução do LPFM em joelhos porcinos: parafusos de interferência, âncoras de titânio com suturas, e tenodese no tendão do músculo adutor, com intuito de avaliar a resistência linear sob tração lateral na mesma inclinação da patela até atingir sua falha no sítio femoral do enxerto.

\section{Materiais e Métodos}

Foi realizado um estudo experimental com 30 joelhos frescos de suínos com idades entre 8 e 9 meses e aproximadamente $110 \mathrm{~kg}$ de peso vivo. Os testes foram realizados à temperatura ambiente e as amostras foram mantidas em solução salina por 300 minutos para manter hidratação adequada das peças antes dos ensaios.

Optamos por utilizar articulações de joelhos suínos, que são anatomicamente semelhantes e têm densidade óssea femoral similar às articulações de joelhos humanos. As articulações de joelhos suínos foram utilizadas como modelo comparativo para fixação no fêmur humano, e estudos anteriores relataram propriedades biomecânicas ${ }^{14-17}$ com resultados similares ${ }^{13}$.

Os joelhos foram dissecados uniformemente. A pele e tecido subcutâneo foram retirados após tenotomia dos tendões patelar e quadriceptal, além de todos os tendões extensores, exceto no grupo 3, sendo fixado o enxerto por tenodese no tendão do adutor. Todas as estruturas periféricas foram seccionadas e retiradas, permanecendo apenas o fêmur que foi utilizado para os testes biomecânicos de fixação do LPFM.

Dividimos os 30 fêmures em 3 grupos de 10, de acordo com os tipos de fixação a serem testados. Realizamos a reconstrução do LPFM com enxertos dissecados dos tendões extensores das patas dos suínos. Foram medidos os comprimentos dos côndilos femorais mediais no plano sagital para padronização das amostras.

Considerando as variações de tamanho que o LPFM nativo apresenta, ${ }^{18}$ utilizamos enxertos com $4 \mathrm{~mm}$ de espessura e $126 \mathrm{~mm}$ de comprimento (30 mm para fixação no fêmur, $40 \mathrm{~mm}$ para fixação no equipamento de tração, e $56 \mathrm{~mm}$ de tendão livre) nos testes dos grupos com parafuso e tenodese em tecidos moles, e tendões com $4 \mathrm{~mm}$ de diâmetro e $192 \mathrm{~mm}$ de comprimento nos testes do grupo das âncoras (0 mm para fixação no fêmur, dois braços de $56 \mathrm{~mm}$ livres e dois braços de $40 \mathrm{~mm}$ para fixação na máquina de tração). Todos os enxertos foram medidos com paquímetro mecânico.

No grupo 1, com fixação por parafuso de interferência de titânio $7 \times 25 \mathrm{~mm}$; um fio Kirschner $1.5 \mathrm{~mm}$ foi usado para encontrar o melhor ponto para túnel femoral. Colocado no local anatômico da inserção do LPFM, podendo ser referenciado pelo epicôndilo femoral medial, $10 \mathrm{~mm}$ proximalmente e $2 \mathrm{~mm}$ posteriormente; ou pelo tubérculo do adutor do fêmur, $4 \mathrm{~mm}$ distalmente e $2 \mathrm{~mm}$ anteriormente. 6,17

Uma broca canulada de $7 \mathrm{~mm}$ é passada sobre o fio até uma profundidade de $30 \mathrm{~mm}$, acomodando as extremidades livres do tendão e não permitindo que o parafuso de $25 \mathrm{~mm}$ ultrapasse a extremidade livre do tendão no túnel garantindo assim uma melhor fixação. ${ }^{19}$ Usando-se um pino de Beath de $2 \mathrm{~mm}$, as extremidades do tendão foram passadas pelo túnel femoral, com os chuleios saindo através do côndilo lateral. Com tração aplicada no chuleio, o enxerto é fixado com um parafuso de interferência de titânio medindo $7 \times 25 \mathrm{~mm}$, e, como na técnica preconizada, a cabeça do parafuso foi enterrada subcorticalmente. ${ }^{6}$

No grupo 2, foi realizado fixação com âncoras de titânio de $5 \mathrm{~mm}$ no fêmur, localizadas no mesmo ponto descrito no grupo anterior; o enxerto foi dobrado em dois braços de comprimentos iguais de $96 \mathrm{~mm}$ e fixado à âncora com fio Ethibond 5.0 (Ethicon Inc., Somerville, NJ, EUA)no centro da dobra sobre o tendão associada a 2 nós com técnica de Pauchet. ${ }^{20} \mathrm{~A}$ tração exercida no teste será em um ângulo de $90^{\circ}$ otimizando a força contra o arrancamento da âncora. ${ }^{21}$

No grupo 3, foi realizada tenodese ao tendão do músculo adutor magno; sendo utilizado fio Ethibond 5.0 através de 4 transfixações da região proximal do enxerto associada a 1 nó com técnica de Pauchet ${ }^{20}$ após cada transfixação, totalizando 4 nós (-Figura $\mathbf{1}$ ).

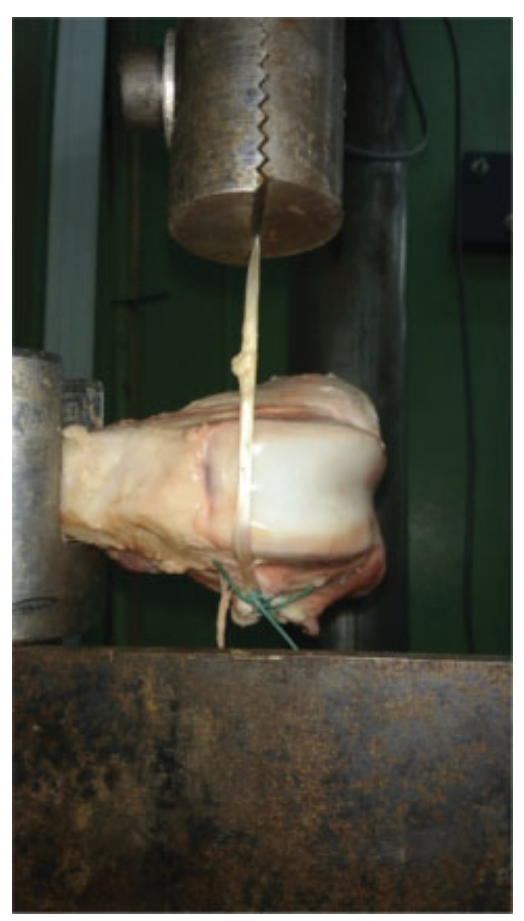

Fig. 1 Visão anterior de fêmur suíno esquerdo com enxerto sendo testado colocado perpendicularmente sob tração na máquina universal de ensaio de tração. Autores, 2017. 
774 Estudo biomecânico de diferentes dispositivos de fixação femoral Dobke et al.

Tabela 1 Resultados da força encontrados e distribuídos conforme o grupo de fixação

\begin{tabular}{|l|l|l|l|}
\hline Grupo & Média (N) & $\begin{array}{l}\text { Desvio } \\
\text { padrão (N) }\end{array}$ & $\begin{array}{l}\text { Coeficiente de } \\
\text { variação (\%) }\end{array}$ \\
\hline 1 Parafuso & $185,45^{(\mathrm{A})}$ & 41,22 & 22,23 \\
\hline 2 Âncora & $152,97^{(\mathrm{B})}$ & 49,43 & 32,31 \\
\hline 3 Tenodese & $76,69^{(\mathrm{C})}$ & 18,90 & 24,64 \\
\hline Valor de $\boldsymbol{p}$ & $\mathrm{p}^{\mathrm{a}}<\mathbf{0 , 0 0 1}^{*}$ & & \\
\hline
\end{tabular}

(*) Diferença estatisticamente significativa a $5 \%$.

${ }^{a}$ Através do teste Kruskal-Wallis com comparações do referido teste. Se as letras subscritas são distintas, se comprova diferença significativa entre os grupos correspondentes.

\section{Testes Biomecânicos}

Testes biomecânicos foram aplicados aos diferentes tipos de fixação do enxerto utilizando uma máquina universal de ensaio de tração com uma velocidade de $20 \mathrm{~mm} / \mathrm{min}$. Eles permitem que a previsão da vulnerabilidade de uma fixação especial venha a fracassar na reabilitação pós-operatória e fornecem um ambiente para a comparação direta de diferentes técnicas e dispositivos de fixação. Os parâmetros de força foram registrados por meio de um sistema de aquisição de dados Spider com 8 canais. O software de processamento de dados utilizado foi o Catman Easy 3.1. Ambos fabricados pela HBM Headquarters Germany/Darmstadt. Os fêmures estavam ligados à base da máquina por um diapasão e paralelos ao solo com o epicôndilo medial voltado para baixo, fixando-os pela sua diáfise por um parafuso ancorado às duas extremidades do diapasão. Um clipe de fixação foi utilizado para fixar a extremidade livre do enxerto à máquina de tração. $O$ enxerto foi mantido na região de fixação femoral em um ângulo de 90 graus com o eixo de tração da máquina (-Figura 1), mantendo a tração visualmente em linha reta com o vetor de deslocamento lateral patelar.

Os parâmetros avaliados foram: a força no limite de resistência máxima dos diversos tipos de fixação do ligamento, expressa em Newtons, e os modos de falhas. Foi definido como falência da amostra quando a curva do gráfico $(\mathrm{N})$ apresentava uma queda súbita durante o teste. 0 teste então era interrompido e o gráfico analisado.

\section{Análise Estatística}

Os dados foram expressos através de média, desvio padrão e coeficiente de variação para as variáveis numéricas e frequências absolutas para a variável categórica.

O tamanho da amostra foi determinado utilizando os métodos de comparação de duas médias (teste $t$ de Student) dos dados de estudos anteriores. ${ }^{13,17}$

Para a comparação entre os grupos em relação a força máxima de tração foi utilizado o teste de Kruskal-Wallis com comparações múltiplas do referido teste. A verificação das hipóteses de normalidade dos dados e de igualdade de variâncias foram realizadas por meio dos testes Shapiro-Wilk e Levene e a utilização das comparações de tamanho foi devido à rejeição da igualdade de variâncias entre os grupos. ${ }^{22}$

A margem de erro utilizada na decisão dos testes estatísticos foi de 5,0\%. Os dados foram digitados na planilha excel e o programa utilizado para obtenção dos cálculos estatísticos foi o SPSS versão 23 (IBM Corp., Armonk, NY, EUA).

Este estudo foi aprovado pela comissão de ética no uso de animais da Universidade(CEUA).

\section{Resultados}

Observou-se que: a média mais elevada de resistência linear sob tração lateral $(185,49 \mathrm{~N})$ ocorreu no grupo 1 (fixação com parafuso), seguido do grupo 2 (fixação com âncora) (152,97 N) e a média menos elevada no grupo 3 (fixação com tenodese) (76,69 N) (-Tabela 1). Para a margem de erro fixada (5\%) se comprova diferença estatisticamente significativa entre os grupos $(p<0,001)$ e através dos testes de comparações múltiplas (entre os pares de grupos) existem diferenças significativas entre eles. A variabilidade expressa pelo coeficiente de variação se mostra reduzida, com medida inferior a 33,3\%. As - Figuras 2, 3 e 4 mostram a força máxima até a falha em cada amostra por grupo.

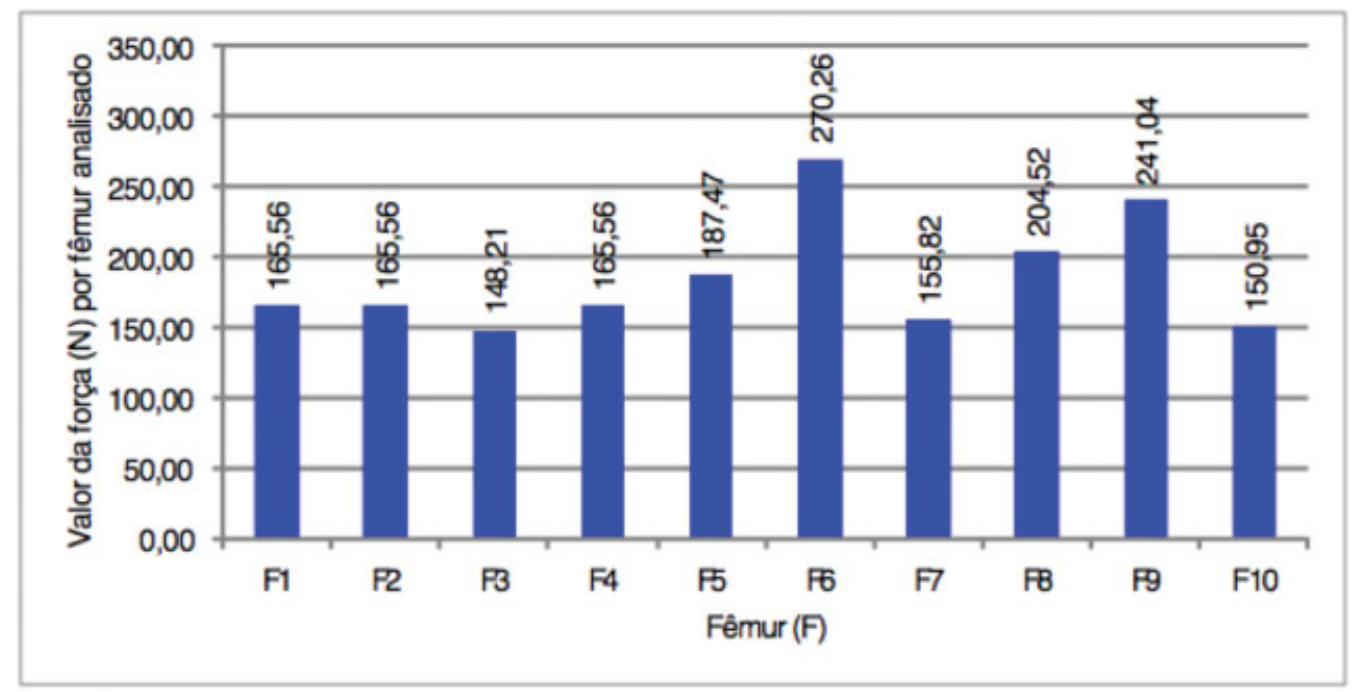

Fig. 2 Valores máximos alcançados da força de tração por fêmur analisado no grupo 1: Fixação por parafuso. Autores, 2017. 


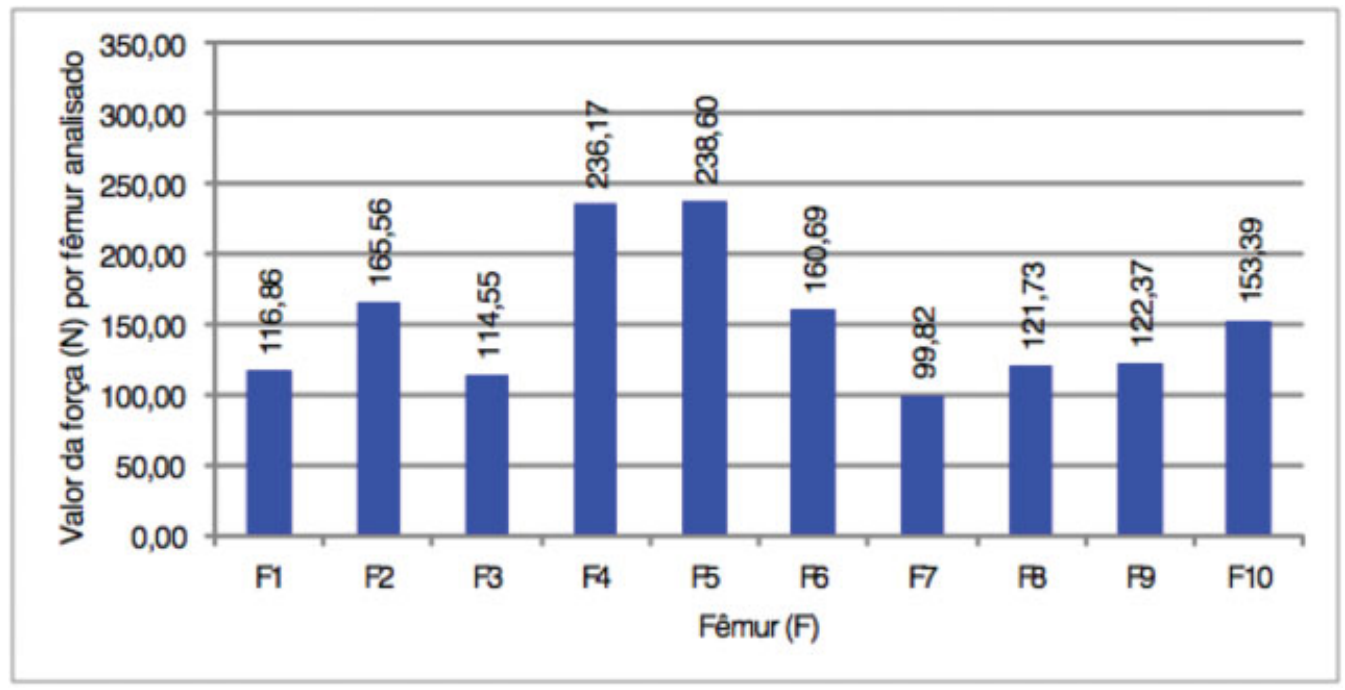

Fig. 3 Valores máximos alcançados da força de tração por fêmur analisado no grupo 2: Fixação por âncora. Autores, 2017.

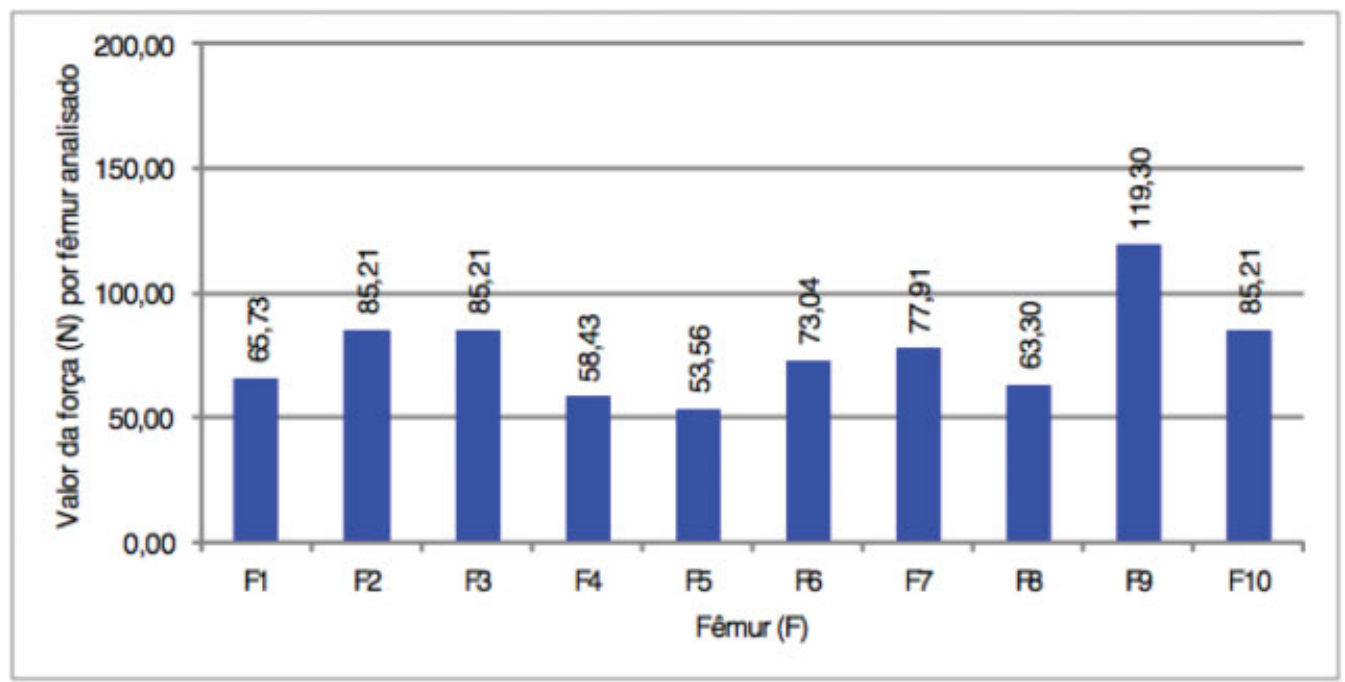

Fig. 4 Valores máximos alcançados da força de tração por fêmur analisado no grupo 3: Fixação por tenodese. Autores, 2017.

Tabela 2 Valores da largura do eixo sagital do côndilo femoral medial distribuídos em cada grupo

\begin{tabular}{|l|l|l|l|}
\hline Grupo & Média (mm) & $\begin{array}{l}\text { Desvio } \\
\text { padrão }(\mathrm{mm})\end{array}$ & $\begin{array}{l}\text { Coeficiente de } \\
\text { Variação (\%) }\end{array}$ \\
\hline 1 Parafuso & 69,00 & 3,09 & 4,48 \\
\hline 2 Âncora & 68,90 & 2,77 & 4,02 \\
\hline 3 Tenodese & 68,40 & 1,78 & 2,60 \\
\hline
\end{tabular}

Houve homogeneidade em relação às larguras no eixo sagital do côndilo femoral em cada grupo. Destacou-se pouca variabilidade entre as peças. Os coeficientes de variação foram no máximo iguais a 4,48\%, como observa-se na - Tabela 2.

Em relação às causas da falha no teste em cada técnica, o grupo 1 (fixação com parafuso) apresentou 6 solturas por deslizamento do enxerto no túnel e as outras 4 falhas foram por ruptura do enxerto; no grupo 2 (fixação por âncora), 8 falhas foram por ruptura do fio de ancoragem e 2 por ruptura
Tabela 3 Tipos de falha distribuídos conforme o tipo de fixação

\begin{tabular}{|l|l|l|l|}
\hline Tipos de falha & Parafusos & Âncora & Tenodese \\
\hline & $\mathrm{N}$ & $\mathrm{N}$ & $\mathrm{N}$ \\
\hline $\begin{array}{l}\text { Deslizamento do } \\
\text { enxerto no túnel femoral }\end{array}$ & 6 & - & - \\
\hline Ruptura do enxerto & 4 & 2 & 10 \\
\hline $\begin{array}{l}\text { Ruptura do fio } \\
\text { de ancoragem }\end{array}$ & - & 8 & - \\
\hline TOTAL & 10 & 10 & 10 \\
\hline
\end{tabular}

(N) = número de amostras.

do enxerto, enquanto que no grupo 3 (fixação por tenodese), todas as 10 falhas foram por ruptura do enxerto ( - Tabela $\mathbf{3}$ ).

\section{Discussão}

O presente trabalho visa comparar os resultados de resistência linear biomecânica dos três métodos de fixação previamente 
descritos. Observou-se que a fixação com parafusos de interferência em túnel ósseo (grupo 1) apresentou a maior carga de resistência à tração $(185,45 \pm 41,22 \mathrm{~N})$ para a reconstrução do ligamento patelofemoral medial conforme a força do ligamento nativo no humano $(145 \pm 68 \mathrm{~N})$, apresentado no estudo de Criscenti et al. $^{2} \mathrm{O}$ grupo de fixação com âncoras (grupo 2) também apresentou uma média de força $(152,97 \pm 49,43 \mathrm{~N})$ até a falha próxima ao valor do estudo supracitado, fato não verificado no grupo de tenodese (grupo 3 ) no tendão do músculo adutor da coxa $(76,69 \pm 18,90 \mathrm{~N})$.

Os resultados evidenciados nesse trabalho são compatíveis com os dados publicados na literatura: a fixação com âncoras ou parafusos de interferência apresentam bons resultados pós-operatórios. $3,5,7,8$

Analisando os tipos de falha ( - Tabela 3 ) resultantes da força máxima até a falência da fixação em cada grupo, mostrou-se que a falha se dava de forma variada dependo do método de fixação, prevalecendo um tipo de falha em cada grupo.

Nosso estudo evidenciou que o grupo 1 apresentou maior força de tração em relação aos demais grupos testados (-Figura 2) e seu tipo de falha, em maior parte (60\%), foi o deslizamento do tendão no túnel.

No mesmo grupo 1, ocorreu ruptura do enxerto em $40 \%$ das amostras. Sabe-se que a simples colocação do parafuso de interferência compromete as propriedades biomecânicas do enxerto, ${ }^{23}$ o que pode ser atenuado ao evitar o hiperdimensionamento do parafuso selecionado.

No grupo 2, em 8 amostras (80\%), houve falha por ruptura do fio de ancoragem (Ethibond 5.0) e uma variação da força de tração com um coeficiente de variação razoavelmente baixo (32,31\%). Supomos que o tipo de fio de ancoragem juntamente com a interface fio/âncora pode influenciar diretamente na força total que a fixação suportará. Este achado é corroborado por um trabalho que mostra que uma ancoragem usando Ethibond 2.0 (Ethicon) é mais frágil quando comparada com Ethibond 5.0. ${ }^{24}$ Sabendo que há fios com resistência muito superior ao Ethibond 5.0, esta técnica mostrou-se bastante resistente a fim de manter a patela em seu trilho troclear. Barber et al. concluíram que algumas âncoras de sutura mais recentes mostraram melhorias significativas nos valores de carga a falha quando comparados com suturas de poliéster trançado. Logo, observa-se que o material de sutura influencia no modo de falha. ${ }^{23,25}$

Outro fator que parece ter contribuído para o não arrancamento da âncora foi o ângulo de tração do enxerto ter sido de aproximadamente $90^{\circ}$ ( Figura 1), evitando assim ângulos mais agudos $\left(<90^{\circ}\right)$ que, por sua vez, facilitam o arrancamento da âncora. ${ }^{21}$

O grupo 3 apresentou menor resistência à tração (—Figura 4), e seu tipo de falha foi $100 \%$ pela ruptura do enxerto, que foi transfixado pelo fio (Ethibond 5.0) para sua biotenodese. Assim, parece que a utilização de sutura transtendão pode fragilizar o mesmo, deixando-o mais vulnerável. Existem trabalhos que mostram certa vantagem em não transfixar o enxerto, ${ }^{26}$ porém ainda necessitamos de mais estudos para esta comprovação.

Comparações diretas entre espécimes humanos são complicadas, sendo difícil de controlar fatores como idade do doador e diferenças de densidade óssea. Portanto, optamos por usar osso suíno, permitindo controlar esses fatores. Este estudo envolveu o teste biomecânico de tempo zero em condições pós-operatórias imediatas, nenhuma comparação histológica foi possível. Portanto, existe a possibilidade de haver diferença entre o estudo in vitro com os resultados de um estudo in vivo, até pela contribuição dos demais estabilizadores estáticos e dinâmicos presentes na articulação patelofemoral. Também não foi realizada a medição do deslizamento do enxerto para reduzir o erro e nem testes de confiabilidade intra/inter observador. ${ }^{11,27}$

\section{Conclusão}

O uso de parafusos de interferência em túnel ósseo em joelhos porcinos é suficientemente forte para fixação femoral na reconstrução do ligamento patelofemoral medial, assim como a fixação com âncoras montáveis com fio de alta resistência . A tenodese no tendão adutor mostrou-se frágil para essa finalidade.

\section{Conflito de Interesses}

Os autores declaram não haver conflito de interesses.

\section{Referências}

1 Gomez JL, Marczyk LR, Cesar PC, Jungblut CF. Reconstrução do ligamento patelofemoral medial: sua indicação na luxação da patela. Rev Bras Ortop 2003;38(1/2):56-66

2 Criscenti G, De Maria C, Sebastiani E, et al. Material and structural tensile properties of the human medial patello-femoral ligament. J Mech Behav Biomed Mater 2016;54:141-148

3 Arendt EA, Moeller A, Agel J. Clinical outcomes of medial patellofemoral ligament repair in recurrent (chronic) lateral patella dislocations. Knee Surg Sports Traumatol Arthrosc 2011;19(11):1909-1914

4 Amis AA, Firer P, Mountney J, Senavongse W, Thomas NP. Anatomy and biomechanics of the medial patellofemoral ligament. Knee 2003;10(03):215-220

5 Fithian DC, Gupta N. Patellar instability: principles of soft tissue repair and reconstruction. Tech Knee Surg 2006;5:19-26

6 Scott WN. Insall \& Scott cirurgia do joelho. $5^{\circ}$ ed. Rio de Janeiro: Elsevier; 2015

7 Placella G, Pace V, Foster P. Reconstruction of the medial patellofemoral ligament reconstruction for patients with recurrent patellar dislocation: review of surgical techniques and tips to achieve successful reconstruction. Ann Transl Med 2016;4(24):540

8 Shah JN, Howard JS, Flanigan DC, Brophy RH, Carey JL, Lattermann C. A systematic review of complications and failures associated with medial patellofemoral ligament reconstruction for recurrent patellar dislocation. Am J Sports Med 2012;40(08):1916-1923

9 Burrus MT, Werner BC, Conte EJ, Diduch DR. Troubleshooting the Femoral Attachment During Medial Patellofemoral Ligament Reconstruction: Location, Location, Location. Orthop J Sports Med 2015;3(01):2325967115569198

10 Yercan HS, Erkan S, Okcu G, Ozalp RT. A novel technique for reconstruction of the medial patellofemoral ligament in skeletally immature patients. Arch Orthop Trauma Surg 2011;131(08):1059-1065

11 Witoński D, Kęska R, Synder M, Sibiński M. An isolated medial patellofemoral ligament reconstruction with patellar tendon autograft. BioMed Res Int 2013;2013:637678

12 Yang DL, Cheon SH, Oh CW, Kyung HS. A comparison of the fixation strengths provided by different intraosseous tendon lengths during anterior cruciate ligament reconstruction: a biomechanical study in a porcine tibial model. Clin Orthop Surg 2014;6(02):173-179 
13 Ayzenberg M, Arango D, Gershkovich GE, Samuel PS, Saing M. Pullout strength of a novel hybrid fixation technique (Tape Locking Screw ${ }^{\mathrm{TM}}$ ) in soft-tissue ACL reconstruction: A biomechanical study in human and porcine bone. Orthop Traumatol Surg Res 2017;103(04):591-595

14 Lee YS, Han SH, Kim JH. A biomechanical comparison of tibial back side fixation between suspensory and expansion mechanisms in trans-tibial posterior cruciate ligament reconstruction. Knee 2012;19(01):55-59

15 Herbort M, Heletta S, Raschke MJ, et al. Accidental perforation of the lateral femoral cortex in ACL reconstruction: an investigation of mechanical properties of different fixation techniques. Arthroscopy 2012;28(03):382-389

16 Posner M, Owens B, Johnson P, et al. Comparison of Pull-out Strength for Different Bone Block Length in a Porcine Anterior Cruciate Ligament Model. Orthop J Sports Med 2014;2(05): 2325967114532762

17 Shen $\mathrm{HC}$, Chang $\mathrm{JH}$, Lee $\mathrm{CH}$, et al. Biomechanical comparison of Cross-pin and Endobutton-CL femoral fixation of a flexor tendon graft for anterior cruciate ligament reconstruction-a porcine femur-graft-tibia complex study. J Surg Res 2010;161(02): 282-287

18 Placella G, Tei M, Sebastiani E, et al. Anatomy of the Medial Patello-Femoral Ligament: a systematic review of the last 20 years literature. Musculoskelet Surg 2015;99(02):93-103

19 Stalder M, Farshad M, Snedeker JG, Meyer DC. Interference screws should be shorter than the hamstring tendon graft in the bone tunnel for best fixation. Knee Surg Sports Traumatol Arthrosc 2013;21(03):584-588

20 Cirino LM. Manual de técnica cirúrgia para a graduação. São Paulo: Sarvier; 2006

21 Sano H, Takahashi A, Chiba D, Hatta T, Yamamoto N, Itoi E. Stress distribution inside bone after suture anchor insertion: simulation using a three-dimensional finite element method. Knee Surg Sports Traumatol Arthrosc 2013;21(08):1777-1782

22 Altman DG. Practical Statistics for Medical Research. London: CRC Press; 1991

23 Barber FA, Herbert MA, Richards DP. Sutures and suture anchors: update 2003. Arthroscopy 2003;19(09):985-990

24 Rupp S, Georg T, Gauss C, Kohn D, Seil R. Fatigue testing of suture anchors. Am J Sports Med 2002;30(02):239-247

25 LaPrade RF, Gilbert TJ, Bollom TS, Wentorf F, Chaljub G. The magnetic resonance imaging appearance of individual structures of the posterolateral knee. A prospective study of normal knees and knees with surgically verified grade III injuries. Am J Sports Med 2000;28(02):191-199

26 Su WR, Chu CH, Lin CL, Lin CJ, Jou IM, Chang CW. The modified finger-trap suture technique: a biomechanical comparison of a novel suture technique for graft fixation. Arthroscopy 2012;28 (05):702-710

27 Kim MK, Na SI, Lee JM, Park JY. Comparison of bioabsorbable suture anchor fixation on the tibial side for anterior cruciate ligament reconstruction using free soft tissue graft: experimental laboratory study on porcine bone. Yonsei Med J 2014;55(03):760-765 